\title{
Effect of selenium supplementation on biomarkers of bone turnover
}

\author{
G. Perri ${ }^{1}$, T. Hill ${ }^{1}$, J.C. Mathers ${ }^{1}$, J. Walsh ${ }^{2}$, F. Gossiel ${ }^{2}$, K. Winther ${ }^{3,4,5}$, J. Frölich ${ }^{3}$, \\ L. Folkestad ${ }^{3,6}$, S. Cold ${ }^{7}$ and R. Eastell ${ }^{2}$ \\ ${ }^{1}$ Human Nutrition Research Centre, Centre for Healthier Lives, Population Health Sciences Institute, Newcastle \\ University, Newcastle upon Tyne, UK, \\ ${ }^{2}$ Sheffield University, Department of Oncology and Metabolism, Metabolic Bone Centre, Sorby Wing, Northern \\ General Hospital, Sheffield, UK, \\ ${ }^{3}$ Department of Endocrinology, Odense University Hospital, Odense, Denmark, \\ ${ }^{4}$ Centre for Diabetes, Academic Specialist Centre, Stockholm, Sweden, \\ ${ }^{5}$ Department of Molecular Medicine and Surgery, Karolinska Institute, Solna, Sweden, \\ ${ }^{6}$ Department of Clinical Research, University of Southern Denmark, Odense, Denmark and \\ ${ }^{7}$ Department of Oncology, Odense University Hospital, Odense, Denmark
}

Selenium is an essential trace element with roles in musculoskeletal health ${ }^{(1,2)}$. Osteoclast inactivation is associated with selenium supplementation in vitro and selenium status is correlated negatively with markers of bone health ${ }^{(3,4)}$. However, the impact of selenium supplementation on bone turnover markers (BTM) has not been studied. This study investigated the effects of selenium supplementation for up to 5 years in older people on BTM including osteocalcin, procollagen type $1 \mathrm{~N}$-terminal propeptide (P1NP), carboxyterminal collagen crosslinks and bone alkaline phosphatase.

490 Danish men and women (60-74 y) were randomised to receive $0,100,200$ or $300 \mu \mathrm{g}$ of selenium daily as selenium-enriched yeast. Plasma selenium concentration was measured using inductively-coupled-plasma mass spectrometry and BTMs were measured using an autoanalyser at baseline, 6 months and 5 years in non-fasted samples. Data were analysed by ANCOVA with polynomial contrasts to investigate the shape of the dose-response relationships. Covariates included: age, body mass index, baseline plasma selenium concentration, baseline BTM, smoking, alcohol, supplement use and medication.

Plasma selenium concentration increased significantly with increasing selenium supplementation at 6 months $(84.1,155.2,212.3$, $258.3 \mathrm{ng} / \mathrm{ml}$ for placebo, 100,200 and $300 \mu \mathrm{g}$ selenium, respectively) $(\mathrm{P}<0.001)$ and remained elevated at 5 years $(88.2,156.4$, 223.8 and 270.9 respectively) $(\mathrm{P}<0.001)$. At 6 months, there was a significant linear decrease in $\mathrm{P} 1 \mathrm{NP}(\mathrm{P}=0.036, \eta 2=0.019)$ with increasing selenium supplementation but this effect was not apparent at 5 years. There was no significant effect of selenium supplementation on any other BTM.

Selenium supplementation reduced P1NP at 6 months but there were no significant effects on other BTM or after 5 years. Since P1NP is a marker of osteoblast function, the fall in PINP with increasing selenium supplementation suggests a reduction in new bone formation 5 . The impact of this change in bone turnover on bone health remains to be determined.

\section{References}

1. Moreno-Reyes R, et al. (2001) J Bone Miner Res 16, 1556-63.

2. Zhang Z, et al. (2014) Biophys Acta 1840, 3246-3256.

3. Hoeg A, et al. (2012) J Clin Endocrinol Metab 97, 4061-70.

4. Beukhof CM et al. (2016) PLOS ONE 11, e0152748.

5. Kuo T \& Chen CH (2017) Biomark Res 5, 18 